\title{
Perfect mirrors and the self-accelerating box paradox
}

\author{
Donald Marolf and Rafael D. Sorkin \\ Physics Department, Syracuse University, Syracuse, NY 13244.
}

(Dated: September 14, 2021)

\begin{abstract}
We consider the question raised by Unruh and Wald of whether mirrored boxes can self-accelerate in flat spacetime (the "self-accelerating box paradox"). From the point of view of the box, which perceives the acceleration as an impressed gravitational field, this is equivalent to asking whether the box can be supported by the buoyant force arising from its immersion in a perceived bath of thermal (Unruh) radiation. The perfect mirrors we study are of the type that rely on light internal degrees of freedom which adjust to and reflect impinging radiation. We suggest that a minimum of one internal mirror degree of freedom is required for each bulk field degree of freedom reflected. A short calculation then shows that such mirrors necessarily absorb enough heat from the thermal bath that their increased mass prevents them from floating on the thermal radiation. For this type of mirror the paradox is therefore resolved. We also observe that this failure of boxes to "float" invalidates one of the assumptions going into the Unruh-Wald analysis of entropy balances involving boxes lowered adiabatically toward black holes. Nevertheless, their broad argument can be maintained until the box reaches a new regime in which box-antibox pairs dominate over massless fields as contributions to thermal radiation.
\end{abstract}

\section{INTRODUCTION}

Black hole entropy, Hawking radiation, and the generalized second law of thermodynamics are central foci of modern research in quantum gravity. Indeed, although the details may differ, the existence of these phenomena is one of the few points on which the various approaches to quantum gravity all seem to agree, including the stringy approach [1, 2,

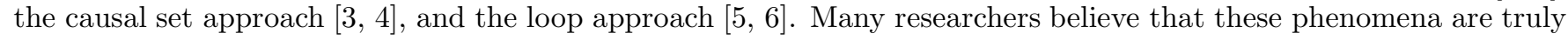
fundamental and that their investigation will reveal further deep laws of nature. Certainly, black hole thermodynamics has provided a fascinating and varied arena for nearly thirty years of research.

One of the most intriguing effects to emerge from this discussion is the experience of thermal radiation by an accelerating object in flat and empty spacetime [7]. This Unruh radiation has a temperature $T$ proportional to the acceleration $a$, and it affects accelerating objects as one would expect, setting off particle detectors and exerting pressure. In a discussion [8] of a potential violation (suggested by Bekenstein [9]) of the generalized second law of thermodynamics, Unruh and Wald pointed out that the pressure on an accelerating box will vary with position, and that the pressure difference across the box must grow quickly with the acceleration. For a perfectly reflecting box of unchanging mass there would therefore be a (box-dependent) critical acceleration $a_{c}$ at which the pressure difference would be big enough to maintain the acceleration without the need for any additional force. From the point of view of the box, it experiences a gravitational field of strength $a_{c}$, but the radiation fluid is sufficiently dense that the mirror simply floats. For a wide but thin box, the critical acceleration will depend only on the mass density (per unit volume) of the box.

Despite appearances, the existence of such an effect would be consistent with energy conservation, from both the inertial and accelerated perspectives [8]. Nevertheless, it is hard to see how it could fail to lead to a disturbing instability of the vacuum and the possibility ${ }^{1}$ of perpetual motion machines. Thus, self-acceleration seems unphysical, and we have referred to this issue in our title as "the self-accelerating box paradox."

We address this paradox in section [1] below, where we consider a specific type of mirror that operates through the use of light internal degrees of freedom. For boxes with mirrored walls of this type, we resolve the self-acceleration paradox by showing that the mirrors necessarily heat up and in doing so acquire enough mass to overwhelm the "buoyant force" on the box, no matter how great the acceleration. The danger of vacuum instability and perpetual motion machines from this quarter thus seems to be removed.

However, it was the "floating box" effect that was used in [8] to argue that the generalized second law could hold without the imposition of novel entropy bounds [9] on matter fields. We therefore reconsider this issue in section III. Although this section is motivated by our study of the self-accelerating box paradox, it constitutes a logically independent discussion that in no way relies on the results of the earlier sections. We find that a derivation very similar to that of [8] can be carried out up to a point (as long as the other assumptions made there are retained).

\footnotetext{
1 As the box passed $a=a_{c}$, the "push" required to keep it accelerating would change into a "pull" (corresponding to a negative net inertial mass). Thus a box held at some $a>a_{c}$ could deliver an infinite amount of work.
} 
However, at depths comparable to what would have been the box's "floating point" the picture changes and one is forced to consider a regime in which "thermal radiation" is dominated by things like box-antibox pairs. This region is a plausible source of effects through which the second law might be maintained, but we are unable to reach a definite conclusion on this question.

\section{WHY MIRRORED BOXES DON'T FLOAT - SOME MODELS AND A CONJECTURE}

We believe that the key to the self-acceleration paradox lies in a study of the mirrors themselves. If this is so, then physical models of the mirrors are needed. Let us therefore consider a mirror that reflects radiation of a certain type, corresponding to excitations of a specific "bulk" field $\phi$.

One can imagine two mechanisms by which such a mirror could function. The first is that the mirror could represent some sort of fundamental potential for $\phi$. This might happen, for example, if the mirror were itself a domain-wall soliton of the field $\phi$, in which case the mass of the linearized perturbations $\delta \phi$ would in general depend on position relative to the wall. Under certain circumstances, the scattering of $\delta \phi$ off of the resulting potential might be strong, making the domain wall act as a good mirror. For lack of a better term, we shall refer to such mirrors as solitonic mirrors in the discussion below.

Note, however, that the mirrors encountered in daily life are decidedly not of this sort. Instead, a standard silver mirror functions by a rather different mechanism. ${ }^{2}$ When a light wave is incident on a silver mirror, the mobile electrons in the mirror rapidly adjust their configuration so as to cancel out the incoming electric field. The result is that the motion of the conduction electrons conspires to send radiation back in the direction from whence the original signal arrived. Because the rapid reconfiguration of these electrons is essential, they necessarily represent "Light Internal Degrees Of Freedom" of the mirror, and we will call mirrors that function along these lines $L I D O F$ mirrors.

Of course, one could also imagine mirrors that operate via a combination of these two mechanisms. One might also ask whether there could be some third mechanism through which a mirror could function. While we are not able to rule out this possibility, it is hard to imagine what this third mechanism might be. In the other direction, one could ask whether one might construe the first mechanism as a special case of the second.

In the rest of this section we shall address the familiar category of LIDOF mirrors and show that they necessarily become heavy when placed in contact with a thermal bath. Due to their many internal degrees of freedom, they will absorb a significant quantity of heat from their environment so that their internal energy and their weight in a gravitational field will both increase with temperature. We will see that this effect is strong enough to overcome any buoyancy force from the radiation fluid, whence the mirror cannot float. Thus, self-acceleration of such a mirror will not occur. (While solitonic mirrors are not addressed in this section, they will be the subject of several comments in section [V.) Although our concern in this section will be with self-acceleration in Minkowski spacetime, we note that almost all of our considerations will apply mutatis mutandis to the case of a (small) box suspended in the vicinity of a black hole.

\section{A. A conjecture and some evidence}

The basis for our analysis of LIDOF mirrors will be the following conjecture:

A perfect LIDOF mirror requires at least one internal field degree of freedom for each bulk degree of freedom that it will reflect. Specifically, the internal degrees of freedom of the mirror must be approximately described as a set of fields in a spacetime of one dimension less than that of the bulk, and these internal fields must have a minimum of one helicity state for each helicity state of the bulk fields.

The emphasis here is on the number of internal degrees of freedom required. ${ }^{3}$ While we have no proof of this conjecture, the following gedankenexperiment provides a certain amount of support.

Consider the scattering of an electromagnetic wave by an electron. One description of this process is that the wave excites the electron, after which the electron re-radiates the wave in a different direction. With enough electrons and enough scattering, one can effectively create a mirror. Now if we naively count degrees of freedom we find two velocity

\footnotetext{
2 Though in the end it might be that the interaction can still be described via an effective, frequency dependent potential.

3 The counting here is of effective degrees of freedom. Such degrees of freedom include, for example, those of weakly interacting quasiparticles describing the excitations of an underlying set of fields or particles which themselves could be strongly coupled.
} 
components for the electron gas, matching the two helicity states of the reflected photons. Moreover, if we imagine that the electrons reside in a superconductor instead of a normal metal, we trade the velocity vector for a scalar "Higgs" field describing the condensate, but since it is a complex scalar, we again recognize two degrees of freedom per point.

Suppose now that the electron couples not just to a single electromagnetic field but to two such fields, EM1 and EM2, and suppose for simplicity that it has the same charge for both. Then our mirror for EM1 will also act as a mirror for EM2. However, consider what happens when a wave of EM1 and a wave of EM2 reach the electrons at the same time. If we arrange for these waves to be exactly opposite in sign, their effects on the electrons will cancel and the electrons will remain undisturbed. As a result, the electrons will not react back on the fields and the waves will pass through the mirror unhindered. Said differently, since the electrons have the same charge for both fields, they couple only to the symmetric combination of EM1 and EM2. The anti-symmetric combination does not interact with the electrons at all, whence the mirror is transparent to its excitations. This would appear to be a general rule: coupling additional gauge fields to a given set of charges results in the charges decoupling from certain linear combinations of the new set of fields, with the result that no more independent linear combinations couple to the charges than originally. Therefore, one cannot violate the conjecture in this way. However, more subtle effects remain to be ruled out.

\section{B. Weight and buoyancy in thermal equilibrium}

We have argued above that a LIDOF mirror must have a significant number of internal degrees of freedom, which can, at least effectively, be described as fields living in the worldvolume of the mirror. Since these mirror fields are responsible for reflecting the bulk fields, they must interact strongly with the latter, and this should be enough to allow them to come into thermal equilibrium with the bulk fields. ${ }^{4}$ Thus, when the mirror is placed in a thermal bath, one would expect the relevant internal degrees of freedom to thermalize on a short timescale, with the result that the mirror will absorb heat if initially it was cold.

A possible objection to this conclusion might be that there might exist types of scattering analogous to the Mössbauer effect. However, we will be considering only boxes whose transverse dimensions are much greater than a wavelength $\lambda$ of the thermal radiation they are reflecting. In this case, it should be possible to think of the radiation as composed of wave packets much smaller than the box wall reflecting them, and causality forbids such a packet from bouncing off the box as a whole. We would thus expect that localized excitations of the relevant internal field must result. (In the opposite case of boxes much smaller than $\lambda$, causality imposes no such restriction, but that case is anyway not amenable to the analysis of this paper, because the fluid approximation we will be using becomes invalid.)

We have argued that the internal degrees of freedom of a perfectly reflecting LIDOF mirror must evolve rapidly to a temperature equal to that of the bath with which they are in contact. We will now use this property to derive a lower bound on the energy density of a perfect mirror in contact with a heat bath. This excess energy is a function of the ambient temperature, and we will show it to be sufficiently great that LIDOF mirrors can never float or self-accelerate.

Given that the mirror helicity states must be in correspondence with the bulk helicity states, it clearly suffices to treat one helicity state at a time, because the full heat absorbed by the mirror is just a sum over mirror helicity states, and the full ambient pressure is a sum over the corresponding bulk helicity states.

In this section we will consider the case of massless fields, though some comments on the massive case will be made in section IV. Moreover, we limit ourselves throughout to free (or almost free) fields, both in the mirror and in the ambient spacetime. (Of course the interaction between bulk and mirror fields is not taken to be weak.) Finally, we work in the adiabatic limit, so that the bulk and mirror fields will always be in equilibrium ${ }^{5}$. Considering a co-dimension one mirror in a $d+1$ dimensional bulk spacetime, we are then left with a rather simple calculation involving the statistical mechanics of a $(d-1)+1$ dimensional massless field and a $\mathrm{d}+1$ dimensional massless field.

Let us first consider the heat absorbed by the mirror. We will take the mirror to be much larger than the ambient thermal wavelength, so that we may use a fluid approximation in computing the energy density of a thermally excited internal field. Notice that, since we are treating this field as a $(d-1)+1$ dimensional system, the thickness of the

\footnotetext{
${ }^{4}$ For a worked out example of such thermalization in a simple case see 33. Notice that no self-coupling of the internal degrees of freedom is involved in this example. Indeed, there is only one such degree of freedom - that of a simple harmonic oscillator - and its self-coupling (nonlinearity) is zero.

${ }^{5}$ More generally our analysis will apply whenever the equilibration time is sufficiently short. If we are right about the rapid thermalization, this is not much of a restriction in practice. In any case, consideration of the adiabatic limit is enough to rule out "floating" selfacceleration, which would be a steady state condition.
} 
mirror will be small compared to the thermal length scale — it is only the lengths along the mirror face that must be large.

In this approximation, the energy density of a $(d-1)+1$ dimensional massless single-helicity bosonic field is given by the expression (see, e.g. [10] for the $3+1$ case):

$$
\begin{aligned}
\rho_{d-1} & =\frac{1}{(2 \pi)^{d-1}} \int d^{(d-1)} k \frac{\omega_{k}}{e^{\beta \omega_{k}-1}} \\
& =\frac{V_{d-2}}{(2 \pi)^{d-1} \beta^{d}} \int_{0}^{\infty} d x \frac{x^{d-1}}{e^{x}-1}
\end{aligned}
$$

where $\beta=1 / T$ and we use units in which Boltzmann's constant and the speed of light are unity. Here, $V_{d-2}=$ $2 \pi^{(d-1) / 2} /((d-3) / 2)$ ! is the volume of the unit $d-2$ sphere and $\omega_{k}$ is the energy of a particle with wavenumber $k$. For our massless field we have, of course, $\omega_{k}=|k|$, and we have used this in the second line above, having also performed the change of variable $x=\beta k$.

Suppose that we wish the mirror to have a proper acceleration $a$, which is related to the ambient temperature through [7] $a=2 \pi / \beta$. Then the mirror needs to experience a co-moving force per unit area of

$$
F / A=a \rho_{d-1}=\frac{V_{d-2}}{(2 \pi)^{d-2} \beta^{d+1}} \int_{0}^{\infty} d x \frac{x^{d-1}}{e^{x}-1} .
$$

Now, what is the actual "buoyant" force per unit area on the box supplied by the ambient thermal radiation? In discussing this question, we will regard as "downward" the direction of the gravitational force felt by the box in its own rest frame. In the case of a box suspended in the vicinity of a black hole horizon this terminology has a literal meaning, but it is also convenient for the case of concern here - that of a box accelerating in a flat background spacetime. Now, the buoyant force is of course just the difference in pressure between the top and bottom of the box, which is bounded above by the ambient pressure on the mirror forming the bottom of the box. ${ }^{6}$ Strictly speaking, this pressure should be calculated from the renormalized stress energy tensor of a quantum field, with appropriate boundary conditions imposed at the walls of the box. However, since such results are not presently available we shall instead follow the approach of [8] and assume that the ambient pressure is in fact equal to that of a thermal fluid in an enclosure of large volume.

Bekenstein has argued [1] that the pressure is somewhat less due to inefficiencies in the scattering of finite wavelength radiation by the box. There may also be other "finite size effects" which are missed in the way we approximate the pressure. ${ }^{7}$ However, since our goal is simply to remove an effect deduced in [8], it is consistent to use their fluid description even if this somehow leads to an overestimate of the ambient pressure.

In this approximation, the pressure (for free fields, as always) is given by an integral much like (2.1) and we have

$$
\begin{aligned}
P_{d} & =\frac{1}{d(2 \pi)^{d}} \int d^{d} k \frac{\omega_{k}}{e^{\beta \omega_{k}-1}} \\
& =\frac{V_{d-1}}{d(2 \pi)^{d} \beta^{d+1}} \int_{0}^{\infty} d x \frac{x^{d}}{e^{x}-1}
\end{aligned}
$$

Our task is now to compare the buoyant force to the weight of the box. From (2.2) and (2.3) their ratio is

$$
\frac{1}{(2 \pi)^{2}} \frac{V_{d-1}}{V_{d-2}} \frac{f(d)}{f(d-1) d}
$$

where we have introduced the definition

$$
f(d)=\int_{0}^{\infty} d x \frac{x^{d}}{e^{x}-1}
$$

\footnotetext{
6 Since this bound ignores the pressure on the top of the box, our analysis applies not only to a box lowered through a region in thermal equilibrium, but also to upward directed thermal radiation of the sort that is present in the Unruh "vacuum" near a black hole.

7 Readers concerned about finite size effects 12 due to the fact that, even in flat spacetime, the scale over which the temperature of the Unruh radiation changes is comparable to a thermal wavelength may consult [13], which provides some evidence that such effects may be ignored. In the corresponding black hole case, there might be further "finite size" effects that we have not accounted for arising from the spacetime curvature.
} 
We claim that this ratio is less than $1 / 4 \pi$ for all $d>1$, reducing in particular to $\pi^{2} / 180 \zeta(3)=0.0456$ for the most important case of $d=3$. In other words the buoyancy is more than an order of magnitude smaller than the weight in all dimensions and more than 20 times smaller in $3+1$-dimensions.

In fact this is not hard to see, using the known formulas

$$
V_{d}=2 \pi^{\frac{d+1}{2}} /\left(\frac{d-1}{2}\right) !
$$

and

$$
f(d)=d ! \zeta(d+1)
$$

where $\zeta$ is the Riemann zeta-function:

$$
\zeta(s)=\sum_{n=1}^{\infty} \frac{1}{n^{s}} .
$$

(The formula for $f(d)$ can be derived by expanding the denominator in (2.5) and noting that $\int_{0}^{\infty} d x x^{n} e^{-x}=n$ !. The formula for $V_{d}$ follows directly from the formula $\pi^{d / 2} /(d / 2)$ ! for the volume of the unit $d$-ball (or "disk"), which in turn can be looked up or proven by induction.)

Substituting this formula for $f(d)$ converts (2.4) into

$$
\frac{1}{(2 \pi)^{2}} \frac{V_{d-1}}{V_{d-2}} \frac{\zeta(d+1)}{\zeta(d)}
$$

and the claimed bound then follows from the obvious inequality $\zeta(d)>\zeta(d+1)$ and the easily checked bound $V_{d} / V_{d-1} \leq \pi$ for $d \geq 1$.

Thus, in all cases we find that the ambient pressure is insufficient to maintain the acceleration of the box.

\section{BLACK HOLES AND THE GENERALIZED SECOND LAW}

Given that a LIDOF mirrored box fails to float in any bath of thermal radiation, it seems worthwhile to reconsider the Bekenstein-Unruh-Wald discussion of whether the laws of thermodynamics, as applied to black holes, impose novel entropy bounds on the matter in the universe (say of the form in [9]). In [8], Unruh and Wald used the floating box effect in analyzing the implications of the generalized second law for a process in which a perfectly reflecting box is lowered adiabatically from infinity to some location near the horizon and then dropped into the black hole. They found that the second law was exactly saturated if the box was dropped just at its floating point. With that choice of release point, the mass contributed to the black hole by the box augmented the black hole's entropy by an amount that exactly offset the disappearance of the entropy contained in the box.

However, since, according to our analysis, the box absorbs heat from the thermal bath and thus increases its weight, one can extract more energy during its descent than in the Unruh-Wald analysis. As a result, by lowering the box adiabatically and releasing it at the point at which the corresponding Unruh-Wald box would float, one might expect to be able to decrease the net entropy of the universe, in contradiction to the generalized second law of thermodynamics. ${ }^{8}$

In opposition to any such scenario, reference [14] points out that if the black hole system can be described as a standard state of thermal equilibrium then no violation of the second law can occur, regardless of the detailed behavior of any perfect mirrors. However, this begs the question of whether the posited description is appropriate and, if it is, of whether the usual expressions for black hole entropy are consistent with this description in the absence of novel entropy bounds. Nor does it tell us what prevents the box from being lowered to its erstwhile floating point as envisioned above with the extraction of more energy than the second law can tolerate.

In the following, we explore to what extent the analysis of [8] can be preserved given the failure of boxes to float. Let us first consider the entropy balance while the box is being quasi-statically lowered. In this phase of the process,

\footnotetext{
8 As usual in these discussions, one means by "entropy of the universe" the sum of all entropy external to the black hole(s) with the entropy belonging to the horizon area of the black holes themselves. In particular, one does not count explicitly the entropy of any object that has fallen through a horizon.
} 
the only change in entropy is that associated with the heat flow from the thermal atmosphere of the black hole into our mirror. Since heat will flow only in a direction that generates entropy, this should create no difficulties with the second law.

It remains to analyze the second stage in which the box is dropped into the black hole. Just before this occurs, the box has some total energy $\epsilon$ and the black hole some total mass $M$. (Here, the energies $\epsilon$ and $M$ are measured relative to the timelike Killing field with unit normalization at infinity.) We remark that $M$ at this stage may be smaller than when the box was far away, due to the energy that flowed from it, through the thermal atmosphere, and into the box during the lowering process. Similarly, $\epsilon$ includes the heat that flowed into the box while it was being lowered along with any other energy that might have entered the box.

Assuming that the box carries no charge or angular momentum, the first law of black hole mechanics tells us that dropping it into the black hole increases the latter's entropy by an amount

$$
\delta S_{b h}=\frac{\epsilon}{T_{H}}=\frac{E}{T}
$$

where $T_{H}$ is the black hole's temperature relative to infinity and $E$ and $T$ are the locally measured energy of the box and locally measured temperature of the thermal radiation. Note that $E$ and $T$ are related to $\epsilon$ and $T_{H}$ by the same redshift factor, which therefore cancels in the ratio (3.1).

The locally measured energy $E$ consists of two pieces. The first, which we shall call $E_{b o x}$, is the energy that the particular internal state of the box would possess in isolation. But, since it requires work $P V$ to bring a box of volume $V$ into the high pressure $(P)$ environment of the thermal atmosphere, the locally measured energy has a second contribution, and is given in full only by the sum $E=E_{b o x}+P V$.

Taking into account the entropy $S_{b o x}$ that disappears into the black hole, the total change in entropy is thus

$$
\delta S=\delta S_{b h}-S_{b o x}=\frac{E}{T}-S_{b o x}=\frac{E_{b o x}+P V}{T}-S_{b o x} .
$$

We now make use of the Gibbs-Duhem relation, $P V=-E_{\text {rad }}+T S_{\text {rad }}$ as in [8], which relates the pressure and volume of thermal radiation to its energy $E_{r a d}$ and entropy $S_{\text {rad }}$. Under the assumption that the radiation constitutes a homogeneous and isotropic fluid whose entropy and energy are additive, this relation can be derived by integrating the first law $P d V=-d E_{r a d}+T d S_{\text {rad }}$ at constant pressure and temperature. ${ }^{9}$ (One is also assuming that the "chemical potential" is zero in the sense that no conservation law freezes the abundance of any constituent of the fluid.) Substituting this result in the above equation, we find

$$
\delta S=\left(\frac{E_{b o x}}{T}-S_{b o x}\right)-\left(\frac{E_{r a d}}{T}-S_{r a d}\right)=\frac{F_{b o x}-F_{r a d}}{T},
$$

where $F_{b o x}$ denotes the Helmholtz free energy of the box and $F_{\text {rad }}$ that of an equal volume of thermal radiation at temperature $T$.

We now recall that thermal radiation minimizes the free energy at fixed temperature ${ }^{10}$. If we assume that the presence of the box does not disturb the free-energy density of the surrounding radiation, and that one can neglect the variation of the latter between the top and bottom of the box, we can conclude that $F_{\text {rad }}<F_{b o x}$ and therefore that $\delta S$ must be positive.

To obtain a physical picture of how this may happen, it is helpful to recall that the free energy of an object controls its probability to occur in a thermal ensemble. In other words, the effective quantum field that describes boxes such as ours will be thermally excited at temperature $T$ just as will any other quantum field near the black hole. When the

\footnotetext{
${ }^{9}$ Near the horizon conditions are far from homogeneous and isotropic. Rather the thermal wavelength of massless radiation is comparable to the scale on which the locally measured temperature varies. For this reason, one may be skeptical of using the Gibbs-Duhem relation in the present context. On the other hand, the results of [13 encourage the belief that the pressure at a certain depth does in fact correspond to that of an unconfined thermal fluid at the appropriately redshifted temperature. As energy conservation relates the density to the change of pressure with depth, the energy density would also be that of an unconfined thermal fluid. This would be enough for the Gibbs-Duhem relation to hold.

10 Of course, this remark would be irrelevant if the "thermal atmosphere" of a black hole were not truly described by a thermal ensemble in this sense, i.e. by a density operator of the Gibbs form $e^{-\beta H}$ which, by the usual arguments of statistical mechanics, is the one that minimizes the free energy at fixed temperature. Evidence that this density operator correctly describes the fields surrounding a black hole comes from the results of e.g. 16, 17, 32] and especially [18, 19, 20, 21, 22, 23] which indicate that the Hawking radiation is described by this density matrix (for the out-going modes). There is also strong evidence from 25], 24] and 17] that any state of the quantum fields in a fixed black hole geometry (and contained in surrounding walls) will evolve toward the Gibbs state possessing the Bekenstein-Hawking temperature of the black hole.
} 
region of space at temperature $T$ is large compared to the size of the box, then $F_{b o x}$ should control the probability for boxes to appear from thermal fluctuations.

What we may conclude from this is not that $F_{b o x}$ is somehow bounded below by some bound set by $F_{\text {rad }}$. Instead, the implication is that, were $F_{b o x}$ to become sufficiently negative, the thermal radiation would consist primarily of box-antibox pairs (as well as related flotsam like fragments of box walls) rather than ordinary massless quanta. Unfortunately, one's understanding of such a "fluid" is minimal, and we have no real grounds on which to judge how well the assumptions we have had to make in the above analysis survive into this new regime. In particular it seems an open question whether anything like the Gibbs-Duhem relation would continue to hold. Thus, at this stage we are unable to demonstrate that the second law is maintained without a better understanding of the "fluid of boxes and box fragments." We will return to these questions in the discussion section.

Notice that the discussion in this section was completely independent of our LIDOF mirror model and should apply to solitonic box walls as well. However, this in itself sheds no light on the extent to which our resolution of the self-acceleration paradox carries over to the case of solitonic mirrors.

\section{DISCUSSION}

In this paper, we have addressed two separate but related issues stemming from consideration of classic thought experiments concerning the generalized second law of thermodynamics. The first was the question of whether perfectly reflecting boxes can self-accelerate in flat spacetime (which in essence is equivalent to the question of whether such boxes can float [8] in the thermal atmosphere of a black hole.) Because the existence of such "runaway solutions" would conflict with cherished beliefs like the stability of the vacuum and the impossibility of a "free lunch", one might expect that they cannot actually occur.

In addressing this paradox, we limited ourselves to mirrors that operate through Light Internal Degrees of Freedom (LIDOF mirrors) and suggested that such mirrors will require a minimum of one internal field degree of freedom (helicity state) for each bulk field degree of freedom (helicity state) to be reflected. ${ }^{11}$ Although we did not prove this assertion, we gave a suggestive example along with supporting arguments.

Accepting this conjecture, we demonstrated that the heat-energy absorbed by such mirrors is more than sufficient to prevent any self-acceleration resulting from the coupling to massless fields in flat spacetime. It is interesting to note that our calculation leaves significant margin for error in the conjecture. For example, in $3+1$ dimensions our conclusions would survive even if the internal degrees of freedom were down by a factor of 20. (It is also clear that one could weaken the claim by a factor of $\sqrt{d}$ and still achieve the desired result. In fact, numerical calculations show that one could weaken the inequality by a factor of the spatial dimension $d$ and that the result would still hold up to $d$ on the order of a few hundred.)

In addition to the question of the validity of our conjectured bound on the number of internal degrees of freedom of a LIDOF mirror, several other questions are raised by our discussion. One of them concerns the other category of mirrors ("solitonic mirrors") and whether one can also derive general constraints forbidding their self-acceleration. While we are not certain how this would work, it seems plausible that a soliton which provides a strong reflecting potential for some field must itself incorporate a strong potential which in turn would force it to have a large tension (and therefore a large mass). Another idea is that the effective description of the soliton in terms of an effective potential might be concealing light internal degrees of freedom which come into play in the course of reflecting external fields. A clear first step in investigating either of these ideas would be to study particular model systems.

A second question concerns the case of massive fields, which are significantly harder to excite when placed in contact with a heat bath. How would the buoyancy compare with the weight if massive fields were the only relevant ones (eg because there were no massless fields in nature or because our mirror was transparent to them)? Of course, if the mass is small compared to the thermal energy $(m \beta \ll 1)$ then such fields will behave as if they were massless. However, for fields with masses higher than the thermal energy scale the situation is rather different. Considering a bulk field of mass $m$ and a mirror field of the same mass ${ }^{12}$, one finds that for $m \beta \gg 1$ the ambient pressure is

$$
P \sim e^{-\beta m} \frac{m^{d / 2}}{\beta^{(d+2) / 2}}
$$

${ }^{11}$ In addition the box wall should have at least one acoustic mode, and this mode should also come into thermal equilibrium with any ambient heat bath, for the same reasons of causality as for the other modes.

12 One expects that a mirror field of greater mass would be unable to adjust sufficiently quickly to reflect the bulk field. On the other hand, making the mirror field lighter than the bulk field would only strengthen our case. 
whereas the heat absorbed by the mirror adds a weight per area of

$$
\frac{W}{A} \sim e^{-\beta m}\left(\frac{m}{\beta}\right)^{(d+1) / 2} \sim P \sqrt{m \beta} \gg P .
$$

We see that, in this case also, the additional weight is more than sufficient to prevent self-acceleration.

Some readers may feel that our analysis does not just place bounds on perfect mirrors, but shows instead that perfect mirrors do not exist at all. They may point out that if the mirror thermalizes with the exterior, it must also radiate thermally into the interior (and vice versa). Thus, the mirror couples the inside with the outside. This is of course true to some extent, but one did not need to count the internal degrees of freedom to make this argument. The relevant question is whether the timescale for this energy flow can be made arbitrarily long. Certainly, our arguments require that a minimum number of degrees of freedom interact with the bulk fields on a short timescale. That they need not interact equally quickly with the interior of the box can be made clear by considering a layered mirror composed of several copies of the simple mirrors studied above. The timescale for transmitting energy between interior and exterior will grow without bound as more and more mirrors are added to the laminate. ${ }^{13}$

In Section [II, we considered the implications of our earlier conclusions for the generalized second law discussions conducted by Bekenstein [9] and Unruh-Wald [8], which historically were the source from which the self-accelerating box paradox arose. Since the main point at issue in those discussions was the relative importance of the buoyant force on a mirrored box, one might think that our results would modify the terms of the debate, and in that way perhaps resolve it.

In the event, however, it seems that the former has occurred without the latter. In particular, we constructed a variant of the derivation of [8] which arrives at similar conclusions without assuming that the box will float. Our derivation is independent of section $\mathbb{I}$ and therefore applies equally well to solitonic mirrors as to LIDOF mirrors. However, we now recognize that the interesting regime is one in which the boxes themselves become an important constituent of the thermal atmosphere.

Let us reflect in more detail on what we have learned from these latter calculations. Some boxes, such as those satisfying the entropy bound $S / E<2 \pi R$ of [9], were long ago established to be 'safe' in the sense that lowering such a box into a black hole respects the second law even when the thermal atmosphere of the black hole is ignored. In our current language such boxes always have $F_{b o x}>0$ since their large size keeps the box far enough from the horizon that we have $T \leq(2 \pi R)^{-1}$. Our calculation once again finds that such boxes are safe and, furthermore, that the existence of such boxes is consistent with any additional assumptions about the black hole's thermal atmosphere so long as $F_{\text {rad }}<0$ (which in turn is equivalent to positivity of the pressure if the Gibbs-Duhem relation holds). The assumption that the atmosphere (and in particular the contributions of boxes and anti-boxes) can be neglected amounts to taking $F_{r a d}=0$.

However, if we imagine a box that violates the above entropy bound then, as it descends toward the horizon and the ambient temperature increases, one may indeed enter a region where $F_{b o x}<0$. In this case, neglect of the thermal atmosphere would lead to an apparent violation of the second law. Similarly, suppose one makes some other assumption as to the nature of $F_{\text {rad }}$. For example, one might assume that $F_{r a d}$ is dominated by the contributions from a certain class $\mathcal{C}$ of states, leading to a relation of the form $F_{\mathcal{C}}=F_{\text {rad }}$. If the Gibbs-Duhem relation holds for this class of states, (3.3) requires the restriction $F_{b o x}>F_{\mathcal{C}}$ for the generalized second law to hold. However, since the free energy controls the relevance of an object or class of states to a thermal ensemble, the existence of a region with $F_{\mathcal{C}}>F_{b o x}$ implies simply that thermal fluctuations in this region include box anti-box pairs in significant numbers and that the class of states $\mathcal{C}$ does not in fact dominate their contribution to the free energy.

When a box enters such a region several new occurrences would seem to be possible which might (or might not) intervene to protect the second law. First, there might be a significant probability that our box would be destroyed by collision with an anti-box. If this happened, we could extract no further energy by trying to lower it adiabatically. Second, with boxes being produced thermally we might observe one being emitted from the black hole - and when combined with the destruction of our own box by an anti-box we might well interpret this as our box "bouncing off" the thermal atmosphere.

Of course, such effects would not be needed if the analysis of Section III could be taken over unchanged to the new regime. The key point here is that if boxes now dominate the thermal radiation, we have $F_{b o x}=F_{\text {rad }}$ as an identity, whence $\delta S$ would vanish identically even if it were somehow meaningful to lower the box to this depth and drop it into the black hole. In this way, one would again maintain the generalized second law without appealing to novel

13 Note, however, that the next few layers of our multi-layer mirror will also interact quickly with the bulk fields and thus absorb even more heat from the thermal bath. 
entropy bounds. However, it is an open question ${ }^{14}$ whether the fluid model we used in Section III could be adequate for computing the "buoyant force" on the box in a domain where boxes (and box fragments) become a dominant component of the thermal radiation. ${ }^{15}$ We used such a model in at least three ways: to justify the "work" term $P V$ in (3.2); to justify using the Gibbs-Duhem relation, which depends on the local homogeneity and isotropy of a fluid; and to justify the assumption that free energy changes are additive when one replaces the box by an equal volume of thermal radiation. ${ }^{16}$

The status of entropy bounds (in the sense of universal bounds that would hold in an arbitrary theory) thus remains elusive. There seems to exist neither a conclusive argument that they must hold nor a conclusive argument to the contrary. ${ }^{17}$ The main contribution of the above analysis in this connection is probably to demonstrate that the question cannot be decided without consideration of a putative new regime in which thermal "radiation" would no longer be dominated by massless fields. ${ }^{18}$ In opposition to earlier suggestions that (in the context of adiabatic box lowering) the second law necessarily fails in the absence of entropy bounds, we have suggested plausible mechanisms for maintaining the second law in this new regime. On the other hand we certainly have not analyzed these mechanisms fully and it would not seem to be an easy task to do so.

The attentive reader may point out that the discussion in [8] showed the second law to be only marginally satisfied when the box was filled with thermal radiation. Since more energy is removed from the system in our scenario than in that of [8], the energy of the box should be less at any height and, if the box is dropped from the putative floating point, less energy would be transferred to the black hole. Thus, one might wonder how we can avoid second law violations for this case. Clearly, to raise this objection one must tentatively assume that the thermal radiation mentioned above does not include significant numbers of box/anti-box pairs.

However, without the inflow of heat into the walls, our box was chosen to be indistinguishable from thermal radiation at the putative floating point. Thus, without this inflow, it is exactly at this point that $F_{b o x}=F_{\text {rad }}$ would first be achieved. The issue just raised may be resolved if the net effect of the heat inflow is to lower the free energy of the box, so that this transition occurs higher in the black hole's thermal atmosphere.

To see that this is the case, we divide the energy $E_{b o x}$ into $E_{0}$, the locally measured energy of the box before it was lowered toward the black hole and $Q$, the total heat that flowed into the box while being lowered. We further write $Q=\int q(z) d z$ where $q(z)$ represents the locally measured heat that flowed into the box between depths $z$ and $z+d z$. Similarly, we divide the entropy of the box into the initial entropy $S_{0}$ present before the box was lowered and the entropy gain $S_{Q}$ resulting from the heat absorbed by the box. The free energy of the box is thus

$$
F_{b o x}=E_{0}-T S_{0}+Q-T S_{Q}
$$

We wish to show $T S_{Q}>Q$ so that $F_{b o x}<E_{0}-T S_{0}$. But since the heat flow occurred without work being done (in particular, without changing the volume of the box), a local application of the first law of thermodynamics yields

$$
T S_{Q}=T \int d z \frac{q(z)}{T(z)}>Q
$$

In the last step we have merely used the fact that the temperature is greatest at the lowest point yet reached by the box (i.e., the current depth) so that $T>T(z)$. Thus we see that for what would have been the marginal case, box/anti-box pairs already dominate the thermal radiation at the would-be floating point.

A final question is whether the results of Section II above might not be germane in analyzing some of the "time machines" that people have tried to imagine. While we know of no direct application, many of the "materials science"

14 Most of these uncertainties relate to "finite size effects" in the broad sense of the term. See for example the comments in 11 about the effects of finite wavelength on the scattering of thermal radiation. And no one who harbored such doubts before is likely to be any happier when they are told that now one is talking about a fluid of box-antibox pairs rather than photons.

15 More generally, the critical question is whether the thermal atmosphere can provide sufficient buoyancy to insure that the work gained by lowering the box (including its mirrored walls) is not so great as to exceed the box's initial entropy appropriately weighted by the black hole temperature.

16 Notice also that, even for more ordinary thermal radiation, the fact that (in the regimes of interest) the box is never more than one or two thermal wavelengths from the horizon calls into question the adequacy of ignoring the inhomogeneities and anisotropies induced by boundary conditions at the box walls.

17 It used to be thought that a world with exponentially many species $N$ of particles/fields could serve as a counterexample (eg [26]), but this also can be questioned, at least in the case of an area bound like $S<A / G$, as it neglects the $N$-dependent renormalization of Newton's constant $1 / G$. As envisioned in connection with entanglement entropy [27] by 28 and 29 this correction would, if the arguments of 28 are correct, more than compensate the contribution of a large $N$ to $S$. See, however, 30 and 31 for comments on the ideas of 28 .

18 Notice that the analysis in Section 11 of whether a mirrored box can self-accelerate/float would also have to be reopened in such a regime. 
issues that arise, for example, in attempts to hold open "wormholes" are similar to those involved in analyzing boxes with impermeable, rigid walls, like those we have been considering in this paper. This suggests that a very general property of such walls, like that conjectured in Section II, could turn out to be important in the "time machine" context as well.

\section{Acknowledgments}

Both authors would like to thank Jacob Bekenstein and Bob Wald for extensive helpful comments on an early draft of this paper and Ted Jacobson for input concerning renormalization issues. D.M. would also like to thank Bob Wald and Eanna Flanagan for numerous conversations about black hole thermodynamics and floating boxes over the past few years. He would also like to thank Abhay Ashtekar and Eric Poisson for clarifying discussions.

D.M. was supported in part by NSF grants PHY97-22362 and PHY00-98747, the Alfred P. Sloan foundation, and by funds from Syracuse University. He would also like to thank the Aspen Center for Physics for their hospitality during part of this work. R.D.S. was partly supported by NSF grant PHY-0098488, by a grant from the Office of Research and Computing of Syracuse University, and by an EPSRC Senior Fellowship at Queen Mary College, University of London. He would also like to express his warm gratitude to Goodenough College, London for providing a splendid living and working environment which greatly facilitated the writing of this paper.

[1] A. Strominger and C. Vafa, "Microscopic origin of the Bekenstein-Hawking entropy," Phys. Lett. B 379 99 (1996) arXiv:hep-th/9601029.

[2] A. Peet, "TASI lectures on black holes in string theory" [arXiv:hep-th//0008241].

[3] D. Dou, "Causal Sets, a Possible Interpretation for the Black Hole Entropy, and Related Topics", Ph. D. thesis (SISSA, Trieste, 1999) gr-qc/0106024.

[4] , Djamel Dou and Rafael D Sorkin, "Is black hole entropy the number of causal links crossing the horizon?" (in preparation)

[5] A. Ashtekar, J. Baez, A. Corichi, and K. Krasnov, "Quantum Geometry and Black Hole Entropy," Phys. Rev. Lett 80904 (1998) arXiv:gr-qc/9710007.

[6] A. Ashtekar, J. Baez, and K. Krasnov, "Quantum geometry of isolated horizons and black hole entropy," Adv. Theor. Math. Phys. 4, 1 (2001) arXiv:gr-qc/0005126.

[7] W. G. Unruh, "Notes on Black Hole Evaporation," Phys. Rev. D 14870 (1976).

[8] W. G. Unruh and R. M. Wald, "Acceleration Radiation And Generalized Second Law Of Thermodynamics," Phys. Rev. D 25, 942 (1982).

[9] J. D. Bekenstein, "Black Holes And Entropy," Phys. Rev. D 7, 2333 (1973); 23, 287 (1981); 49, 1912 (1994).

[10] K. Huang, Statistical Physics (Wiley, NY, 1987).

[11] J. D. Bekenstein, "Non-Archimedean character of quantum buoyancy and the generalized second law of thermodynamics," Phys. Rev. D 60, 124010 (1999) arXiv:gr-qc/9906058.

[12] L. Li and L. Liu, "Properties of radiation near the black hole horizon and the second law of thermodynamics," Phys. Rev. D46 3296, (1992).

[13] W. Anderson, "The Boulware State and the Generalized Second Law of Thermodynamics," Phys. Rev. D50 4786 (1994) arXiv:gr-qc/9402030.

[14] S. Gao and R. M. Wald, "The 'physical process' version of the first law and the generalized second law for charged and rotating black holes," Phys. Rev. D 64, 084020 (2001) arXiv:gr-qc/0106071.

[15] M.A. Pelath and R. M. Wald, "Comment on entropy bounds and the generalized second law," Phys. Rev. D 60, 104009 (1999) arXiv:gr-qc/9901032.

[16] Hawking, S. W. "Particle Creation by Black Holes," Comm. Math. Phys. 43 199-220.

[17] K. Fredenhagen and R. Haag, "On The Derivation Of Hawking Radiation Associated With The Formation Of A Black Hole," Commun. Math. Phys. 127, 273 (1990).

[18] W. G. Unruh and N. Weiss, "Acceleration Radiation In Interacting Field Theories," Phys. Rev. D 29, 1656 (1984).

[19] R. Laflamme, "Geometry And Thermofields," Nucl. Phys. B 324, 233 (1989).

[20] A. O. Barvinsky, V. P. Frolov and A. I. Zelnikov, "Wave Function Of A Black Hole And The Dynamical Origin Of Entropy," Phys. Rev. D 51, 1741 (1995) arXiv:gr-qc/9404036.

[21] T. Jacobson, "A Note On Hartle-Hawking Vacua," Phys. Rev. D 50, 6031 (1994) arXiv:gr-qc/9407022.

[22] T. A. Jacobson, "Introduction to Black Hole Microscopy," arXiv:hep-th/9510026.

[23] T. Jacobson, "Comment on *Accelerated detectors and temperature in (anti) de Sitter spaces*," Class. Quant. Grav. 15, 251 (1998) arXiv:gr-qc/9709048.

[24] R.D. Sorkin, "The Statistical Mechanics of Black Hole Thermodynamics", in R.M. Wald (ed.) Black Holes and Relativistic Stars, (U. of Chicago Press, 1998), pp. 177-194 arXiv:gr-qc/9705006.

[25] J. Bisognano and E. Wichmann, "On the duality condition for a Hermitian scalar field", J. Math. Phys. 16: 985 (1975)

[26] R.D. Sorkin, R.M. Wald and Zhang Zh.-J., "Entropy of Self-Gravitating Radiation", Gen. Rel. Grav. 13:1127-1146 (1981) 
[27] L. Bombelli, R.K. Koul, Lee J. and R.D. Sorkin, "A Quantum Source of Entropy for Black Holes", Phys. Rev. D 34, 373383 (1986). R.D. Sorkin, "On the Entropy of the Vacuum Outside a Horizon", in B. Bertotti, F. de Felice and A. Pascolini (eds.), Tenth International Conference on General Relativity and Gravitation (held Padova, 4-9 July, 1983), Contributed Papers, vol. II, pp. 734-736 (Roma, Consiglio Nazionale Delle Ricerche, 1983)

[28] L. Susskind and J. Uglum, "Black hole entropy in canonical quantum gravity and superstring theory", Phys. Rev. D 50:2700-2711 (1994) hep-th/9401070

[29] T. Jacobson, "Black Hole Entropy and Induced Gravity", [gr-qc/9404039]

[30] S. N. Solodukhin, "One loop renormalization of black hole entropy due to nonminimally coupled matter," Phys. Rev. D 52, 7046 (1995) arXiv:hep-th/9504022.

[31] D. Kabat, "Black hole entropy and entropy of entanglement," Nucl. Phys. B 453, 281 (1995) arXiv:hep-th/9503016; D. Kabat, S. H. Shenker and M. J. Strassler, "Black hole entropy in the O(N) model," Phys. Rev. D 52, 7027 (1995) arXiv:hep-th/9506182.

[32] R.D. Sorkin, "A Simplified Derivation of Stimulated Emission by Black Holes", Classical and Quantum Gravity 4, L149L155 (1987)

[33] A.O. Caldeira and A.J. Leggett, Physica A121: 587 (1983) and 130: 374(E) (1985) 